\title{
Pulmonary Lipoma in an Atypical Location of the Pulmonary Fissure, Extirpated by Uniportal VATS - Case Report and Review of Literature
}

Authors:

Disclosure:

Received:

Keywords:

Citation:
Daniel Bulyashki 1,2 *Zarina Brady, ${ }^{1}$ Shahswar Arif, ${ }^{1}$ Radoslav Radev, ${ }^{1,2}$ Nikolay Cvetkov, ${ }^{1,2}$ Hristo Popov ${ }^{3}$

1. Medical University "Prof. Dr. Paraskev Stoyanov", Varna, Bulgaria

2. Clinic of Thoracic Surgery, University Hospital "St Marina" Varna, Bulgaria

3. Department of General and Clinical Pathology, Forensic Medicine and Deontology, University Hospital "St. Marina", Varna, Bulgaria Correspondence to zarinadb@hotmail.com

The authors have declared no conflicts of interest.

14.09.2019

02.01 .2020

Lipoma, pleural cavity, pulmonary fissure, thoracoscopy.

EMJ Respir. 2020;DOI/10.33590/emirespir/19-00168

\section{Abstract}

Lipomas are common benign tumours characterised by soft moveable masses throughout various locations in the body. However, atypical localisation of the pulmonary fissure is uncommon. Herein is reported a rare case of a female with an asymptomatic lesion in the pulmonary fissure of the left lung extracted by single-port video-assisted thoracoscopic surgery, with a good clinical and radiologic outcome. Single-port video-assisted thoracoscopic surgery technique with a brief review of relevant literature will be discussed.

\section{INTRODUCTION}

transferred for further diagnostic approach due to a mass in the left lung found on routine chest adults and can manifest in all parts of the body. Although they are of high frequency, intrathoracic involvement is rare. Herein a case is presented with atypical localisation of lipoma in the fissure of the left lung. To the best of the authors' knowledge, this is the first known successful treatment of a pulmonary lipoma by single-port video-assisted thoracoscopic surgery (VATS) in Bulgaria.

\section{CASE REPORT}

A 56-year-old Bulgarian female patient was evaluating the adipose tumour preoperatively, admitted to the authors' hospital in June 2018 with no metabolic uptake seen (Figure 1C).
Using uniport VATS, a $4 \mathrm{~cm}$ incision was made in the fifth intercostal space between the anterior and the midaxillary line. A well-circumscribed, yellowish mass measuring approximately 6-7 $\mathrm{cm}$ was visualised in the fissure of the left lung located next to the pulmonary artery (Figure 2), and was removed (Figure 3). Histological examination confirmed the diagnosis of lipoma (Figure 4), and no malignancy was noted. The patient was discharged on the fourth day after surgery, and had an uneventful postoperative period, with very slight need of analgesic drugs. A 6-month follow-up X-ray revealed no evidence of recurrence (Figure 1D).

\section{DISCUSSION}

Lipomas are the most common benign mesenchymal tumours in adults, accounting for $5-6 \%$ of all lung tumours.,2 Approximately $90 \%$ of benign lung tumours are adenomas or hamartomas. Pulmonary lipomas consist mainly of mature adipose tissue and are rarely found in the thorax. ${ }^{2,3}$ It is known that smoking and obesity are significant risk factors for endobronchia lipomas, and account for $0.1-0.5 \%$ of all lung tumours. 2,4 Intrathoracic lipomas can be located in the mediastinum, bronchial tree, pulmonary parenchyma, and pleura. Recurrent symptoms of persistent cough, dyspnoea, fever, chest pain and wheezing can be noted in the majority of lipomas, making differential diagnosis wide 5 The full extent of obstruction determines the level of symptoms and the required surgical intervention Mediastinal lipomas are slow progressing, thus symptoms may not be severe until larger tumour growth compresses adjacent structures.

Malignancy can be seen in tumours that involve the tracheobronchial tree,? with the majority of endobronchial lipomas presenting in the first three subdivisions on the right. ${ }^{4}$ Lipomas arising from the pleura are commonly seen in the lower section of the cavity. ${ }^{8}$ Their origin is most commonly of the left posterolateral side of diaphragmatic substance. Plural lipomas are most often slow rrowing and patients remain asymptomatic unti routine chest X-ray. ${ }^{5}$ Current literature grossly lacks information on pulmonary lipomas with lung parenchymal involvement, especially in such a are asymptomatic anatomical location as the left pulmonary fissure, as well as possible treatment approaches; thus, this case report serves as an important learning point to educate the medical community.

ntrathoracic lipomas are classified into two groups according to Keeley and Vana, ${ }^{9}$ and Williams and Parsons: ${ }^{10}$ 1) dumbbell shaped lipomas that move through the thoracic inlet or intercostal space; and 2) solely intrathoracic lipomas.11-13 Additionally, intrathoracic lipomas can be classified according to their origin 1) endobronchial lipoma arising from the subcutaneous fat of the tracheobronchial tree; 2) parenchymal lipoma found peripherally in the lung parenchyma; 3) pleural lipoma arising from the submesothelial parietal pleura and can reach subpleural, pleural, or extrapleural spaces; 4) mediastinal lipoma; and 5) cardiac lipoma." Endobronchial lipomas, usually located in the submucousal layer of the bronchial walls, are thought to be derived from the fatty tissue in the walls. The fatty content in the bronchial wall decreases with the progressive branching of the bronchi and is not seen in bronchioli smaller than $1 \mathrm{~mm}$ in diameter. This may partly explain why peripheral intrapulmonary lipomas are extremely rare. Giant pulmonary lipomas, i.e., those that reach at least 1,000 grams or $10 \mathrm{~cm}$ in diameter, present as round pale yellowish masses that are well-circumscribed and thinly encapsulated. ${ }^{14}$ Usually lipomas are solitary and are not associated with other extrathoracic lesions. They effect both sides with the same frequency of occurrence.

Differential diagnosis varies and includes benign ung tumours, malignant lung tumours, infectious granulomas, and noninfectious granulomas. Physicians might include adenoma, pulmonary ymphoma, tuberculosis, and sarcoidosis as potential diagnoses during workup investigations. Patients with peripheral intrapulmonary lipoma are asymptomatic because of the lesion location, and they are often found accidentally in routine chest radiographic examination. Some of the patients may have nonspecific symptoms as a result of the ability of the lipoma to develop within the chest cavity and reach a large size. It may provoke symptoms such as mild chest pain intermittent cough, dyspnoea, or a sensation of thoracic tightness. For example, clinical disease manifestation can be misconstrued in the case of endobronchial lipomas, falsely indicating obstructive lung diseases, resulting in prolonged diagnosis, insufficient treatment, and excessive costs. 

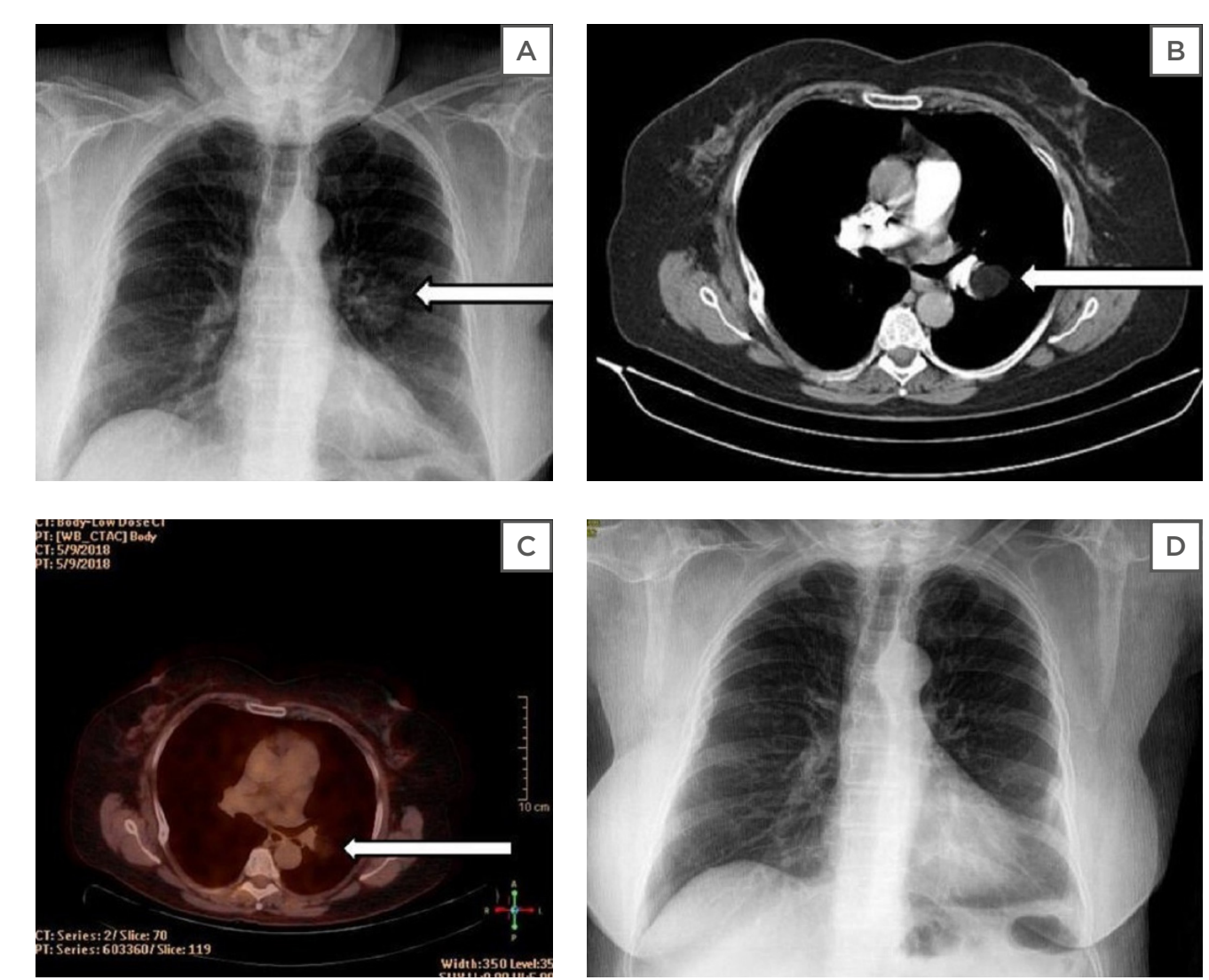

Figure 1: Preoperative and postoperative view.

(A) Chest X-ray frontal view. Preoperative X-ray, arrow showing a well circumscribed mass in the pulmonary fissure of the left lung. (B) Preoperative axial CT scan lung window. Arrow showing a homogeneous well-defined lowdensity mass, measuring $32 \times 26 \times 62 \mathrm{~mm}$, located in the pulmonary fissure of the left lung. The lesion appears to be approximately 93 (HU). (C) Preoperative axial FDG-PET fusion window. Arrow showing a non-enhanced, low-density mass noted in the pulmonary fissure of the left lung. (D) Postoperative chest X-ray frontal view. Six-month follow-up chest $\mathrm{X}$-ray frontal view, finding no residual mass.

DG: fluorodeoxyglucose; HU: Hounsfield units.

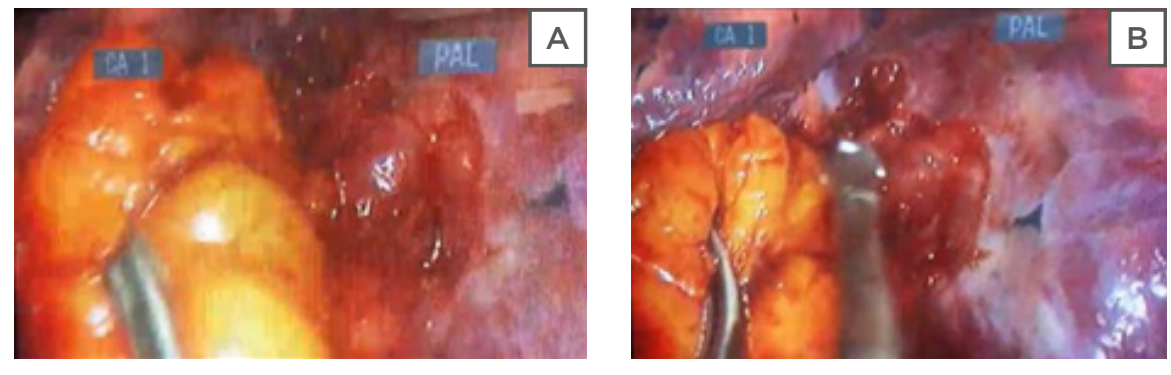

Figure 2: (A; B) Intraoperative mass visualised in the pulmonary fissure. Well circumscribed, yellowish mass seen in an atypical location of the pulmonary fissure, using Ethicon Harmonic."

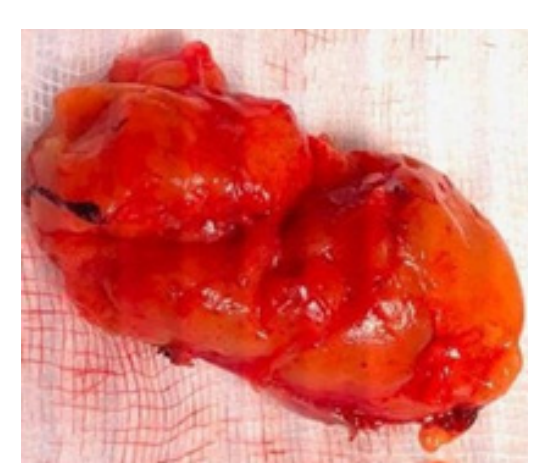

Figure 3: Gross specimen. Gross appearance of the pulmonary lipoma; a well-circumscribed, soft, lobulated, yellow mass.
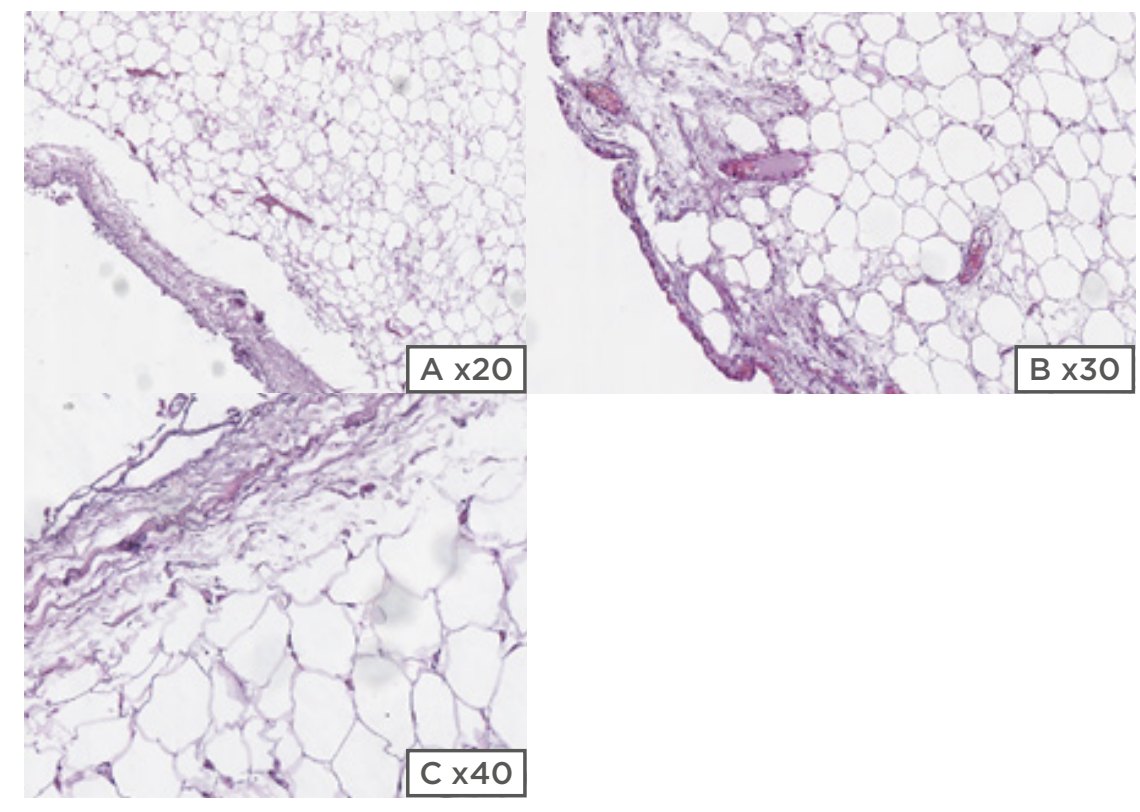

Figure 4: (A; B; C) Histology of pulmonary lipoma. Benign neoplasm composed of mature adipose tissue. Slide prepared with haematoxylin and eosin.

Parenchymal lipomas could be mistaken for pneumonia in patients presenting with fever and soft tissue opacity on chest X-ray.

On a plain chest radiograph, lipomas present as smooth, well-defined masses and allow for visualisation of structural compression. It is very common to incidentally uncover pulmonary lipomas in asymptomatic patients upon routine radiograph, a very cost-effective occurrence for both the patient and the healthcare system. The ability to differentate between benign and The ability to differentiate between benign and malignant processes, as well as distinguish plain chest X-ray is very limited. Chest CT with by pathological examination, which should

the value of -50 to $-150 \mathrm{HU}$ and a well-defined differentiate between these two tumours, benign homogeneous hypodense mass may suggest the diagnosis of lipoma. ${ }^{15}$ Moreover, such scans provide essential information to differentiate between pleural and parenchymal disease, as well as help determine the location and extent of disease. Contrast enhanced MRI may provide more detailed analysis of the lipoma fatty density, its heterogeneity, and its relationship with contiguous organs. FDG-PET-CT may be useful preoperatively for differentiation between benign lesions and liposarcoma, with enhancing areas indicating malignancy. ${ }^{16}$ 
lesions, and liposarcoma. Surgical resection can easily be performed via an open typical or muscle-sparing thoracotomy. Complete excision should be performed to prevent recurrences. In the last two decades, VATS has become the most common technique for thoracic tumour operations. In the management strategy for pulmonary lipoma cases we may also include clinical and radiological follow-up for elderly patients and those in a poor general condition.

\section{CONCLUSION}

Although pulmonary lipomas are considered a very rare tumour, it requires attention, skill, and knowledge to differ lipoma from liposarcoma. VATS constitutes a valuable, minimally invasive surgical procedure for establishing a firm diagnosis and complete excision of lesions.

\section{Key Take-home Message}

When presented with such a future patient, a chest CT should be the initial investigation of choice, while MRI and PET-CT are useful for preoperative planning and indicating malignancy. Differential diagnosis can be fast tracked by having a solid understanding and correlating the location of the lesion with fat characteristics. In comparison to other techniques, VATS offers significant advantages in better control of postoperative pain, lung expansion, and decreased morbidity and mortality rates. However, is it important to highlight this procedure is contraindicated in haemodynamically unstable patients, and in this case an open thoracotomy is indicated. The major teaching point of this case is to highlight to physicians, surgeons, and medical students that thorough knowledge and investigation is indispensable due to this disease expressing features of other pulmonary conditions; thus, accurate diagnosis can be challenging.

\section{References}

1. Naeem M et al. Giant lipoma chest extending into the thoracic cavity unique entity: a case report. J Med Case Rep. 2017:1(3):131-4.

2. Pollefliet $\mathrm{C}$ et al. Endobronchial lipomas: rare benign lung tumors, two case reports. Thorac Oncol. 2009:4(5):658-60.

3. Sivapalan P et al. An obstructing endobronchial lipoma simulating COPD. Eur Clin Respir J. 2014;12:1.

4. Muraoka M et al. Endobronchial lipoma: review of 64 cases reported in Japan. Chest. 2003:123(1):293-6.

5. Gaerte et al. Fat-containing lesions of the chest. Radiographics. 2002:22:S61-78.

6. Chen $\mathrm{CH}$ et al. A rare case of dysphagia and palpitation caused by the compression exerted by an enormous mediastinal lipoma. Rev Port Pneumol. 2012:18(3):149-52.

7. Dy RV et al. Endobronchial lipoma causing progressive dyspnea. Respir Med Case Rep. 2017:22:95-7.

8. Sureka B et al. Radiological review of pleural tumors. Indian J Radiol Imaging. 2013:23(4):313-20.

9. Keeley JL, Vana AJ. Lipomas of the mediastinum; 1940 to 1955 . Surg Gynecol Obstet. 1956;103(4):313-22.

10. Williams WT, Parsons WH. Intrathoracic lipomas. J Thorac Surg. 1957;33(6):785-90.

11. Parsons L et al. Intraparenchymal pulmonary lipoma: pathologicradiologic correlation of a rare presentation of a common neoplasm. Ann Diagn Pathol. 2014:18(4):244-7.
12. Surani S et al. Endobronchial lipomatous polyp: a rare benign tumor of the lung. Case Rep Pulmonol. 2014:240834.

13. Badescu $\mathrm{C}$ et al. Unusual thoracic Lipoma; a case report and literature review. Iran J Radiol. 2018:15(4):e61304.

14. Lang CL et al. Gigantic subcutaneous lipoma - a case report. JPRAS Open. 2015;3(1):6-9.

15. Zhu CM et al. Intrapulmonary lipoma: a case report and literature review. World J Pediatr. 2015:11(2):185-7.

16. Suzuki R et al. PET evaluation of fatty tumors in the extremity: possibility of using the standardized uptake value (SUV) to differentiate benign tumors from liposarcoma. Ann Nucl Med. 2005;19(8):661-70. 